Inna Belfer · Gabriel Phillips · Julie Taubman

Heather Hipp · Robert H. Lipsky · Mary-Anne Enoch

Mitchell B. Max · David Goldman

\title{
Haplotype architecture of the norepinephrine transporter gene SLC6A2 in four populations
}

Received: 13 January 2004/ Accepted: 13 February 2004/Published online: 9 April 2004

(C) The Japan Society of Human Genetics and Springer-Verlag 2004

\begin{abstract}
The norepinephrine transporter (NET) regulates levels of monoamine neurotransmitters integral to a variety of behaviors and autonomic functions. Two SLC6A2 polymorphisms have been used in genetic association studies, generating intriguing but nondefinitive results on traits such as hypertension and mood. One of these SLC6A2 variants is functional but rare. The other is common but not informative over the entire $48 \mathrm{~kb} S L C 6 A 2$ region and is insufficient to capture the functional diversity potentially contained within any SLC6A2 region. To elucidate SLC6A2 haplotype structure and define markers sufficient to capture haplotype diversity within detected haplotype blocks, 26 single-nucleotide polymorphisms (SNPs) were genotyped in 384 individuals evenly divided across Finnish Caucasian, US Caucasian, Plains American Indian, and African American populations. Three conserved blocks, $13.6,12.5$, and $25 \mathrm{~kb}$ in size and showing little evidence for historical recombination were observed in all populations. Haplotype diversity in block 1 and numbers of common haplotypes were highest in African Americans, among whom 5-6 optimal markers were sufficient to maximize diversity of each block. For other populations, 2-3 markers/block sufficed, but the optimal markers
\end{abstract}

Parts of this work were presented at the 53rd Annual Meeting of American Society of Human Genetics, Los Angeles, CA, USA, November 2003.

I. Belfer · G. Phillips · H. Hipp · M. B. Max

Pain and Neurosensory Mechanisms Branch,

National Institute of Dental and Craniofacial Research,

Bethesda, MD, USA

I. Belfer $(\varangle) \cdot$ G. Phillips $\cdot$ J. Taubman $\cdot$ H. Hipp ·

R. H. Lipsky · M.-A. Enoch · D. Goldman

Laboratory of Neurogenetics,

Department of Health and Human Services,

National Institute on Alcohol Abuse and Alcoholism,

National Institutes of Health, Bethesda, MD

20892-1258, USA

E-mail: ibelfer@mail.nih.gov

Tel.: + 1-301-402-8323

Fax: + 1-301-443-8579 differed across populations. The SLC6A2 haplotype map and 25-marker panel (excluding the monomorphic one) is a comprehensive tool for genetic linkage studies on phenotypes related to NET function.

Keywords Single-nucleotide polymorphism - Linkage disequilibrium $\cdot$ Haplotype $\cdot$ Norepinephrine transporter $\cdot S L C 6 A 2$

\section{Introduction}

In humans, norepinephrine (NE) is essential to fundamental cognitive and emotional processes including attention, learning and memory, perception of emotions, and perception of pain (Foote et al. 1983; Jasmin et al. 2002). NE is also involved in autonomic control via its actions in the brainstem and as the primary neurotransmitter at postganglionic sympathetic nerve terminals (Hahn et al. 2003). The majority of brain noradrenergic neurons are concentrated in the locus coeruleus, a phylogenetically ancient and developmentally precocious structure. These NE neurons project to limbic regions critical to cognition and affect.

NE released at central and peripheral synapses is inactivated through active transport into terminals by the presynaptically localized norepinephrine transporter (NET) (Iversen 1974). NET recaptures as much as $90 \%$ of released NE making it a critical mediator of NE inactivation and presynaptic catecholamine homeostasis (Schomig et al. 1989). Thus, NET plays a role in controlling the intensity and duration of signal transduction (Zahniser et al. 2001). NE interacts with many other neurotransmitters both in normal cortical regulation and in the therapeutic response to psychoactive compounds, and one critical interacting neurotransmitter is dopamine (Jordan et al. 1994). Dopamine is the NE precursor so that levels of both neurotransmitters are regulated by common factors, for example, tyrosine 
Table 1 Primer and probe sequences for $5^{\prime}$ nuclease genotyping of $26 S L C 6 \mathrm{~A} 2$ single-nucleotide polymorphisms (SNPs)

\begin{tabular}{|c|c|c|}
\hline SNP & Primers and probes & Sequences \\
\hline \multirow[t]{4}{*}{1} & Forward primer & AAGGTCATCTCATAACCTAGCAGAGA \\
\hline & Reverse primer & TCCCTAAGACGCAACTCTAGCA \\
\hline & Allele 1 probe (FAM) & CACAGAGCTCCATGTGG \\
\hline & Allele 2 probe (VIC) & CAGAGCCCCATGTGG \\
\hline \multirow[t]{4}{*}{2} & Forward primer & GGACAACAAAGCACAAAGAGTGT \\
\hline & Reverse primer & GCTTGTGTGGCCAGGATGT \\
\hline & Allele 1 probe (FAM) & TCAGCTGTTTTAATGAAG \\
\hline & Allele 2 probe (VIC) & CAGCTGTTTTGATGAAG \\
\hline \multirow[t]{4}{*}{3} & Forward primer & GCTTGTGTGGCCAGGATGT \\
\hline & Reverse primer & CCAGACAAGTGCCAGCTCTT \\
\hline & Allele 1 probe (FAM) & CAGAGGGAGACTCCT \\
\hline & Allele 2 probe (VIC) & AGAGGGAGGCTCCT \\
\hline \multirow[t]{4}{*}{4} & Forward primer & AAGGCACTGATACACGTAGTCTCTA \\
\hline & Reverse primer & AAACCCATTCATGAACACTTAGTTCTGA \\
\hline & Allele 1 probe (FAM) & CTCCCGATCCACTGTAG \\
\hline & Allele 2 probe (VIC) & CCCGATCCGCTGTAG \\
\hline \multirow[t]{4}{*}{5} & Forward primer & GATCATAGATACACCCACCTCCСТTA \\
\hline & Reverse primer & AGTTCTGAATGCTTTCTTCCATGAA \\
\hline & Allele 1 probe (FAM) & ATGCTACGAATTATG \\
\hline & Allele 2 probe (VIC) & AATGCTACAAATTATG \\
\hline \multirow[t]{4}{*}{6} & Forward primer & TCCGACCTCATGAACCTAGCTT \\
\hline & Reverse primer & CTGTCCCCGCTGTTTTGG \\
\hline & Allele 1 probe (FAM) & CTCTCCCACTTTGG \\
\hline & Allele 2 probe (VIC) & TCTCTCTCACTTTGGG \\
\hline \multirow[t]{4}{*}{7} & Forward primer & GGTTCAGAACACATGGCTTGAGA \\
\hline & Reverse primer & CTGCAGGGAATCAGGAAAGC \\
\hline & Allele 1 probe (FAM) & CCCACAAGTACCTCCAC \\
\hline & Allele 2 probe (VIC) & CACAAGTGCCTCCAC \\
\hline \multirow[t]{4}{*}{8} & Forward primer & CCTCACCCAGCTCCATCCT \\
\hline & Reverse primer & GGAGACTGTGCTGGAAGTTCGT \\
\hline & Allele 1 probe (FAM) & CATGCAGGGCTTC \\
\hline & Allele 2 probe (VIC) & ACATGCAGGACTTC \\
\hline \multirow[t]{4}{*}{9} & Forward primer & CCCAGGTGACCCAGAGC \\
\hline & Reverse primer & CCCAGGTGACCCAGAGC \\
\hline & Allele 1 probe (FAM) & TTTCCTTCTCGCCCTGTT \\
\hline & Allele 2 probe (VIC) & СТТТССТТСТСССССТGTT \\
\hline \multirow[t]{4}{*}{10} & Forward primer & AGTTTCCGGTGTCGCTTCAG \\
\hline & Reverse primer & CCAGATGGGAGGCATGGA \\
\hline & Allele 1 probe (FAM) & CAGGCCCGTGATGA \\
\hline & Allele 2 probe (VIC) & AGGCCTGTGATGACA \\
\hline \multirow[t]{4}{*}{11} & Forward primer & TGAACACTAGGCCAGCTCCTGTA \\
\hline & Reverse primer & GCAGCTCAGACCAATGGTTTC \\
\hline & Allele 1 probe (FAM) & AAACAGCAAGGACC \\
\hline & Allele 2 probe (VIC) & AGAAACATCAAGGACC \\
\hline \multirow[t]{4}{*}{12} & Forward primer & TGCTTCCTGAACTGAGCTAGATCAT \\
\hline & Reverse primer & GCCTGGCAGGGTCAGAAGT \\
\hline & Allele 1 probe (FAM) & ТСТССССТGCAGTCT \\
\hline & Allele 2 probe (VIC) & AGTCTCCCTTGCAGTC \\
\hline \multirow[t]{4}{*}{13} & Forward primer & САСТАССАААТТСАСССАСТТТСАС \\
\hline & Reverse primer & CССCAАCACCCTCATCCA \\
\hline & Allele 1 probe (FAM) & CAGAGGCCCCAACT \\
\hline & Allele 2 probe (VIC) & AGGCCCAAACTAT \\
\hline 14 & Forward primer & AATCAGGCTAAGGTCAAGGTAGTCA \\
\hline & Reverse primer & CCCCCTTAGGGATATGGGAAGATT \\
\hline & Allele 1 probe (FAM) & TTCAGGTGAGTCCTC \\
\hline & Allele 2 probe (VIC) & TGTTTCAGCTGAGTCC \\
\hline 15 & Forward primer & TCACTTTAAATCCATACCTGCTGGTA \\
\hline & Reverse primer & GCCATTGGCTTGAATTTGGTA \\
\hline & Allele 1 probe (FAM) & ACCACACAAATTAT \\
\hline & Allele 2 probe (VIC) & CCACACAGATTATC \\
\hline 16 & Forward primer & CTTAGAGATTGCTGTAACTGAAACAAAAA \\
\hline & Reverse primer & AAAGTCTAGATAATAAGAGTTCATTGCACAA \\
\hline & Allele 1 probe (FAM) & TCCCAAGAAACAGCT \\
\hline & Allele 2 probe (VIC) & TCCCAAGAGACAGCT \\
\hline 17 & Forward primer & GAGGCCCTTGGCAGTATCC \\
\hline & Reverse primer & AGTTCCTCATATTTTCCTGCCAAA \\
\hline & Allele 1 probe (FAM) & CCTGGTCTCTGTTATT \\
\hline & Allele 2 probe (VIC) & TGGTCTCTGCTATTAG \\
\hline
\end{tabular}


Table 1 (Continued)

\begin{tabular}{|c|c|c|}
\hline SNP & Primers and probes & Sequences \\
\hline \multirow[t]{4}{*}{18} & Forward primer & GGCTCCAGGCCAGAAAGG \\
\hline & Reverse primer & GGCCTCTGCAAGTAGATAGGAGTTC \\
\hline & Allele 1 probe (FAM) & AGGGCGGCATGAG \\
\hline & Allele 2 probe (VIC) & AGGGCAGCATGAGA \\
\hline \multirow[t]{4}{*}{19} & Forward primer & GCTCTTCCCTTCTCTGACATCATC \\
\hline & Reverse primer & GCGTGGGTAGTCAGGAAAGG \\
\hline & Allele 1 probe (FAM) & AGGTGACATAGGAACT \\
\hline & Allele 2 probe (VIC) & AGGTGACGTAGGAAC \\
\hline \multirow[t]{4}{*}{20} & Forward primer & CTGAAACACAAGAAGAGCGATAGATT \\
\hline & Reverse primer & GGGATTGGCTTCAGGTATAGATAGAT \\
\hline & Allele 1 probe (FAM) & CCAGACATCATTATTT \\
\hline & Allele 2 probe (VIC) & CAGACAACATTATTTG \\
\hline \multirow[t]{4}{*}{21} & Forward primer & TACAGGTGGCTGAAGCAAAGG \\
\hline & Reverse primer & GGACCCCATCAAGTTTCTGTGA \\
\hline & Allele 1 probe (FAM) & CAGGGAAGTTACCC \\
\hline & Allele 2 probe (VIC) & AGGGAAGTCACCCAC \\
\hline \multirow[t]{4}{*}{22} & Forward primer & CACTTTGGCCAGTTTCCTCAAC \\
\hline & Reverse primer & AACTGGGCAGTTTACCTATGTGTTG \\
\hline & Allele 1 probe (FAM) & AAACCCCTGGGTGAC \\
\hline & Allele 2 probe (VIC) & TAAACCTCTGGGTGACAC \\
\hline \multirow[t]{4}{*}{23} & Forward primer & CATGACCCAAACACСТСТСАСТАG \\
\hline & Reverse primer & TGTCTCCACTAAAACTCCTGTTGAAG \\
\hline & Allele 1 probe (FAM) & CACTCCCACACTGG \\
\hline & Allele 2 probe (VIC) & ACTCCCAAACTGG \\
\hline \multirow[t]{4}{*}{24} & Forward primer & AGCACACTGAGGGCTGTTAAATAAC \\
\hline & Reverse primer & СCTGGCTCAGGGTTACACTGA \\
\hline & Allele 1 probe (FAM) & CTGGGTAAGTCCTGG \\
\hline & Allele 2 probe (VIC) & TGGGTGAGTCCTGG \\
\hline \multirow[t]{4}{*}{25} & Forward primer & CCAAACAGCCATGCTTAAACCT \\
\hline & Reverse primer & AGCTAATCCAGAGGTGAAACTTTCC \\
\hline & Allele 1 probe (FAM) & AGTAACGCCTGAGAGG \\
\hline & Allele 2 probe (VIC) & AACGCTTGAGAGGC \\
\hline \multirow[t]{4}{*}{26} & Forward primer & TCСТАСССАТССТССАААACC \\
\hline & Reverse primer & GGTTATCAGGGAAGGCTTCACA \\
\hline & Allele 1 probe (FAM) & CCAGTCACCCTCAC \\
\hline & Allele 2 probe (VIC) & TCCAGTCAACCTCA \\
\hline
\end{tabular}

hydroxylase activity. The NET has the ability to transport dopamine, and drugs that block the NET increase extracellular levels of both NE and DA (Tanda et al. 1997; Bymaster et al. 2002; Gu et al. 1996). Monoamine transporters are initial sites of action for several antidepressant drugs (including several which are relatively NET selective) as well as psychostimulants including cocaine and the amphetamines (Pacholczyk et al. 1991; Ritz et al. 1990; Tatsumi et al. 1997; Sacchetti et al. 1999). Decreases in NE uptake sites and activity have been observed in hypertension, diabetes, cardiomyopathy, and heart failure (Esler et al. 1981; Merlet et al. 1992; Bohm et al. 1995; Schnell et al. 1996; Backs et al. 2001), and insufficient NE clearance may contribute to the progression of these diseases (Bohm et al. 1998).

The human NET gene (SLC6A2, hCG2025341) is located on chromosome 16q12.2 (Brüss et al. 1993) and has 15 exons spanning $\sim 48 \mathrm{~kb}$ (Pörzgen et al. 1995, 1998). The cDNA sequence encodes a 617 -amino acid protein with 12 highly hydrophobic membrane domains and a high level of amino acid identity to other members of the $\mathrm{Na}^{+} / \mathrm{Cl}^{-}$-dependent monoamine transporter family, e.g., $H T T$ (serotonin transporter) and $D A T$ (dopamine transporter) (Nelson 1998; Hahn and Blakely 2002). SLC6A2 has five alternative splice transcripts. Resequencing of SLC6A2identified 13 DNA sequence variants, among them five low-frequency missense substitutions (Stober et al. 1996). The reported missense substitutions Val69Ile, Thr99Ile, Val245Ile, Val449Ile, and Gly478Ser are located in putative transmembrane domains 1, 2, 4, 9, and 10, respectively. The Thr99Ile substitution is at the 5 th position of a putative leucine zipper in transmembrane domain 2. A rare Ala457Pro substitution in exon 9 resulting in more than $98 \%$ loss of function has recently been detected (Ivancsits et al. 2003). A synonymous substitution also located in exon 9. A $1287 \mathrm{G}$, has been used in a series of association studies to NE-related phenotypes including hypertension and mood disorders (Stober et al. 1996; Leszczynska-Rodziewicz et al. 2002; Samochowiec et al. 2002). However, no common functional NET polymorphisms are known.

The NET markers used so far in linkage studies do not capture the potential information on NET functional variation, and the results obtained so far have been nondefinitive. A haplotype approach combining abundant missense polymorphisms with a series of loci 
chosen for haplotype informativeness offers the potential for detection of effects of any allele of moderate abundance and effect size, regardless of whether the allele is presently known or unknown (Gabriel et al. 2002). Concerning linkage disequilibrium (LD), many regions of the genome have a block-like structure such that all loci within the block region tend to be in strong LD. However, haplotype block boundaries, strength of LD, haplotype diversity, and optimal marker panels to fully capture haplotype diversity vary across populations. In this study, we report the haplotype structure of SLC6 A2obtained using 26 single-nucleotide polymorphisms (SNPs) genotyped in four populations: Finnish and American Caucasians, American Indians, and African Americans. We also describe marker panels for each block, which maximize haplotype information content.

\section{Materials and methods}

Participants A total of 384 participants were genotyped, including 96 individuals from each of four populations: Finns, US Caucasians, African Americans, and Plains American Indians. Informed consent was obtained according to human research protocols approved by the human research committees of the recruiting institutes, including the National Institute on Alcohol Abuse and Alcoholism, National Institute of Mental Health, Rutgers University, and University of Helsinki. All participants had been psychiatrically interviewed, and none had been diagnosed with a psychiatric disorder.

SNP markers The physical position and frequency of minor alleles $(>0.05)$ from a commercial database (Celera Discovery System, CDS, July, 2003) were used to select SNPs (including A1287G and Ala457Pro). A total of 50 SLC6A2 SNPs were identified in the database. $5^{\prime}$ nuclease assays (vide infra) could be designed for 35 , and of these, 26 SNP assays detected sequence polymorphisms and could be genotyped in highly accurate fashion. This panel of 26 equally spaced markers covered the $48-\mathrm{kb}$ gene plus $4 \mathrm{~kb}$ upstream and $4 \mathrm{~kb}$ downstream.

Genomic DNA Genomic DNA was extracted from lymphoblastoid cell lines and diluted to a concentration of $10 \mathrm{ng} / \mathrm{ul}$; 1-ul aliquots were dried in 384-well plates.

Table 2 Locations and allelic frequencies of 26 SLC6A2 SNPs in 96 individuals from each of four populations ${ }^{\mathrm{a}}$. \# Single-nucleotide polymorphism (SNP) marker with location shown in Fig. 1. Orientation is \#26-\#1 $\left(5^{\prime}-3^{\prime}\right)$

\begin{tabular}{|c|c|c|c|c|c|c|c|c|c|}
\hline \# & $\begin{array}{l}\text { SNP ID } \\
\text { (CDS) }\end{array}$ & $\begin{array}{l}\text { SNP ID } \\
\text { (NCBI) }\end{array}$ & Variation & $\begin{array}{l}\text { Position } \\
\text { (CDS) }\end{array}$ & Location & \multicolumn{4}{|c|}{ Allelic frequency (for allele 2) } \\
\hline 1 & hCV1232486 & rs258099 & $\mathrm{T}>\mathrm{C}$ & 42997914 & $3^{\prime}$ intergenic & 0.67 & 0.71 & 0.68 & 0.17 \\
\hline 2 & hCV1232479 & rs171798 & $A>G$ & 43000649 & 3 intergenic & 0.82 & 0.78 & 0.88 & 0.46 \\
\hline 3 & hCV1232477 & rs7188230 & $\mathrm{A}>\mathrm{G}$ & 43001386 & $3^{\prime}$ intergenic & 0.81 & 0.76 & 0.97 & 0.43 \\
\hline 4 & hCV26354929 & no rs & $\mathrm{A}>\mathrm{G}$ & 43003652 & $3^{\prime}$ intergenic & 0.16 & 0.27 & 0.02 & 0.34 \\
\hline 7 & hCV26354922 & rs8049681 & $\mathrm{A}>\mathrm{G}$ & 43009804 & Intron 14 & 0.13 & 0.16 & 0.02 & 0.38 \\
\hline 8 & hCV1232472 & rs 1800887 & $\mathrm{G}>\mathrm{A}$ & 43011565 & Intron 11 & 0.83 & 0.73 & 0.97 & 0.47 \\
\hline 9 & Ala457Pro & no rs & $\mathrm{G}>\mathrm{C}$ & 43013237 & Exon 9 & 1.00 & 1.00 & 1.00 & 1.00 \\
\hline 10 & hCV3020068 & rs5569 & $\mathrm{C}>\mathrm{T}$ & 43013319 & Exon 9 & 0.27 & 0.38 & 0.44 & 0.11 \\
\hline 11 & hCV 1232470 & rs5568 & $\mathrm{G}>\mathrm{T}$ & 43015030 & Intron 7 & 0.62 & 0.66 & 0.77 & 0.86 \\
\hline 12 & hCV1232469 & rs1861647 & $\mathrm{C}>\mathrm{T}$ & 43016748 & Intron 6 & 0.31 & 0.40 & 0.45 & 0.12 \\
\hline 18 & hCV3020077 & rs 1875544 & $\mathrm{G}>\mathrm{A}$ & 43033100 & Intron 4 & 0.25 & 0.34 & 0.37 & 0.09 \\
\hline 19 & hCV1232444 & rs 187714 & $\mathrm{~A}>\mathrm{G}$ & 43038757 & Intron 4 & 0.29 & 0.42 & 0.46 & 0.25 \\
\hline 20 & hCV7719239 & rs933555 & $\mathrm{T}>\mathrm{A}$ & 43040802 & Intron 3 & 0.73 & 0.59 & 0.55 & 0.74 \\
\hline 21 & hCV1232442 & rs41154 & $\mathrm{T}>\mathrm{C}$ & 43042550 & Intron 2 & 0.28 & 0.42 & 0.47 & 0.23 \\
\hline 22 & hCV1232440 & rs6499771 & $\mathrm{C}>\mathrm{T}$ & 43044584 & Intron 2 & 0.82 & 0.82 & 0.84 & 0.84 \\
\hline 23 & hCV1232433 & rs 36028 & $\mathrm{C}>\mathrm{A}$ & 43048097 & Intron 2 & 0.21 & 0.15 & 0.08 & 0.18 \\
\hline 24 & hCV1232429 & no rs & $A>G$ & 43050568 & Intron 2 & 0.87 & 0.85 & 0.86 & 0.95 \\
\hline 25 & hCV1232426 & rs36031 & $\mathrm{C}>\mathrm{T}$ & 43052435 & Intron 2 & 0.71 & 0.59 & 0.53 & 0.67 \\
\hline 26 & hCV1232422 & rs4783899 & $\mathrm{C}>\mathrm{A}$ & 43058059 & $5^{\prime}$ intergenic & 0.34 & 0.46 & 0.48 & 0.37 \\
\hline
\end{tabular}

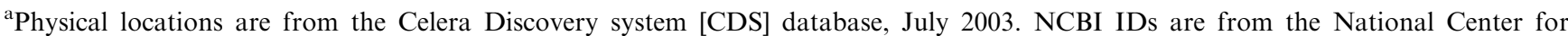
Biotechnology Information database, November 2003 


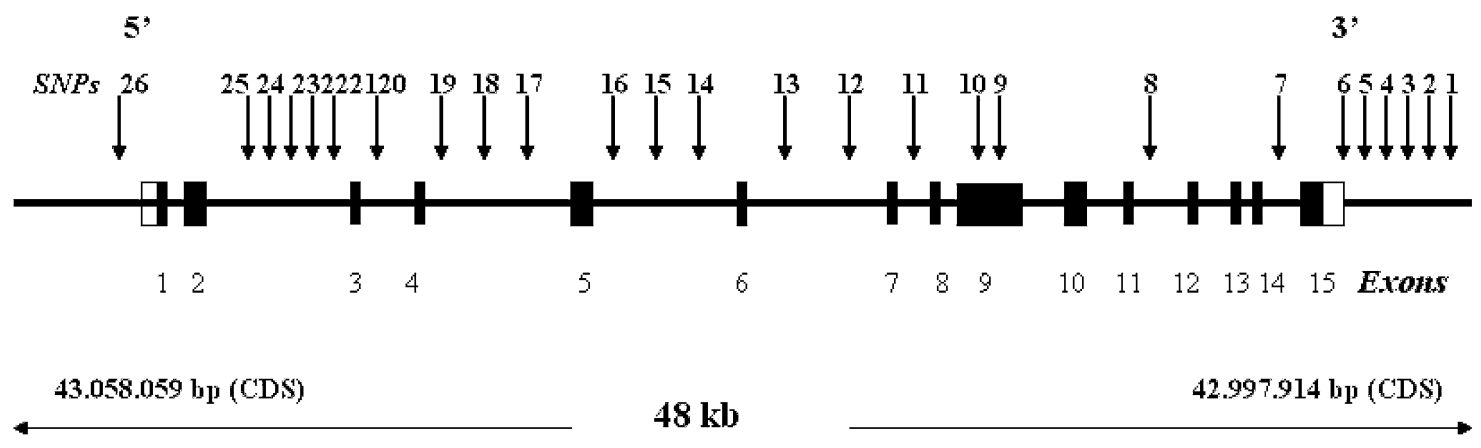

Fig. 1 Location of single-nucleotide polymorphisms (SNPs) genotyped in the human SLC6A2 gene. Coding exons are shown as solid blocks; $5^{\prime}$ and $3^{\prime}$ UTRs are indicated by unfilled rectangles. Physical locations are from the Celera Discovery System [CDS] database, July 2003

Polymerase chain reaction (PCR) amplification Genotyping was performed by the $5^{\prime}$ nuclease method (Shi et al. 1999) using fluorogenic allele-specific probes. Oligonucleotide primer and probe sets were designed based on gene sequence from the CDS, July 2003. Primers and detection probes for each locus are listed in Table 1.

In each reaction well, $2.5 \mu \mathrm{l}$ of PCR Master Mix (Applied Biosystems, CA, USA) containing AmpliTaq Gold DNA Polymerase, dNTPs, gold buffer, and $\mathrm{MgCl}_{2}$ was mixed with $900 \mathrm{nmol}$ of each forward and reverse primer and $100 \mathrm{nmol}$ of each reporter and quencher probe. DNA was allowed to stand at $50^{\circ} \mathrm{C}$ for $2 \mathrm{~min}$ and at $95^{\circ} \mathrm{C}$ for $10 \mathrm{~min}$, amplified by 40 cycles at $95^{\circ} \mathrm{C}$ for $15 \mathrm{~s}$ and $60^{\circ} \mathrm{C}$ for $1 \mathrm{~min}$, and then held at $4^{\circ} \mathrm{C}$. PCR was carried out with a GeneAmp PCR system 9700 (Applied Biosystems).

Allele-specific signals were distinguished by measuring endpoint 6-FAM or VIC fluorescence intensities at 508 and $560 \mathrm{~nm}$, respectively, and genotypes were generated using Sequence Detection System Software Version 1.7 (Applied Biosystems). Genotyping error rate was directly determined by regenotyping $25 \%$ of the samples, randomly chosen, for each locus. The overall error rate was $<0.005$. Genotype completion rate was 0.98 .
Table 3 Pairwise linkage disequilibrium $\left(D^{\prime}\right)$ among eight single-nucleotide polymorphisms (SNPs) in haplotype block 1 across four populations

\begin{tabular}{|c|c|c|c|c|c|c|c|c|}
\hline U.S. Caucasians marker & 1 & 2 & 3 & 4 & 5 & 6 & 7 & 8 \\
\hline 1 & & 1.00 & 0.66 & 0.66 & 0.65 & 0.60 & 0.60 & 0.66 \\
\hline 2 & & & 0.71 & 0.71 & 0.70 & 0.66 & 0.66 & 0.71 \\
\hline 3 & & & & 1.00 & 1.00 & 1.00 & 1.00 & 1.00 \\
\hline 4 & & & & & 0.86 & 1.00 & 1.00 & 1.00 \\
\hline 5 & & & & & & 1.00 & 1.00 & 0.86 \\
\hline 6 & & & & & & & 1.00 & 1.00 \\
\hline 7 & & & & & & & & 1.00 \\
\hline 8 & & & & & & & & \\
\hline Finnish Caucasians marker & 1 & 2 & 3 & 4 & 5 & 6 & 7 & 8 \\
\hline 1 & & 1.00 & 0.64 & 0.55 & 0.27 & 0.30 & 0.30 & 0.54 \\
\hline 2 & & & 0.79 & 0.79 & 0.34 & 0.37 & 0.37 & 0.82 \\
\hline 3 & & & & 1.00 & 0.82 & 0.86 & 0.86 & 1.00 \\
\hline 4 & & & & & 0.95 & 1.00 & 1.00 & 1.00 \\
\hline 5 & & & & & & 1.00 & 1.00 & 0.95 \\
\hline 6 & & & & & & & 1.00 & 1.00 \\
\hline 7 & & & & & & & & 1.00 \\
\hline 8 & & & & & & & & \\
\hline Plains Indians marker & 1 & 2 & 3 & 4 & 5 & 6 & 7 & 8 \\
\hline 1 & & 1.00 & 0.40 & 0.25 & 0.00 & 0.00 & 0.00 & 0.40 \\
\hline 2 & & & 0.32 & 1.00 & 1.00 & 1.00 & 1.00 & 0.32 \\
\hline 3 & & & & 0.74 & 1.00 & 1.00 & 1.00 & 1.00 \\
\hline 4 & & & & & 1.00 & 1.00 & 1.00 & 0.74 \\
\hline 5 & & & & & & 1.00 & 1.00 & 1.00 \\
\hline 6 & & & & & & & 1.00 & 1.00 \\
\hline 7 & & & & & & & & 1.00 \\
\hline 8 & & & & & & & & \\
\hline African Americans marker & 1 & 2 & 3 & 4 & 5 & 6 & 7 & 8 \\
\hline 1 & & 1.00 & 1.00 & 1.00 & 0.88 & 1.00 & 1.00 & 1.00 \\
\hline 2 & & & 0.81 & 0.92 & 0.83 & 0.93 & 0.93 & 0.81 \\
\hline 3 & & & & 1.00 & 0.82 & 1.00 & 0.93 & 0.75 \\
\hline 4 & & & & & 0.88 & 0.97 & 0.97 & 1.00 \\
\hline 5 & & & & & & 0.87 & 0.87 & 0.87 \\
\hline 6 & & & & & & & 0.97 & 0.97 \\
\hline 7 & & & & & & & & 1.00 \\
\hline 8 & & & & & & & & \\
\hline
\end{tabular}


Table 4 Pairwise linkage disequilibrium $\left(D^{\prime}\right)$ among eight single-nucleotide polymorphisms (SNPs) in haplotype block 2 across four populations

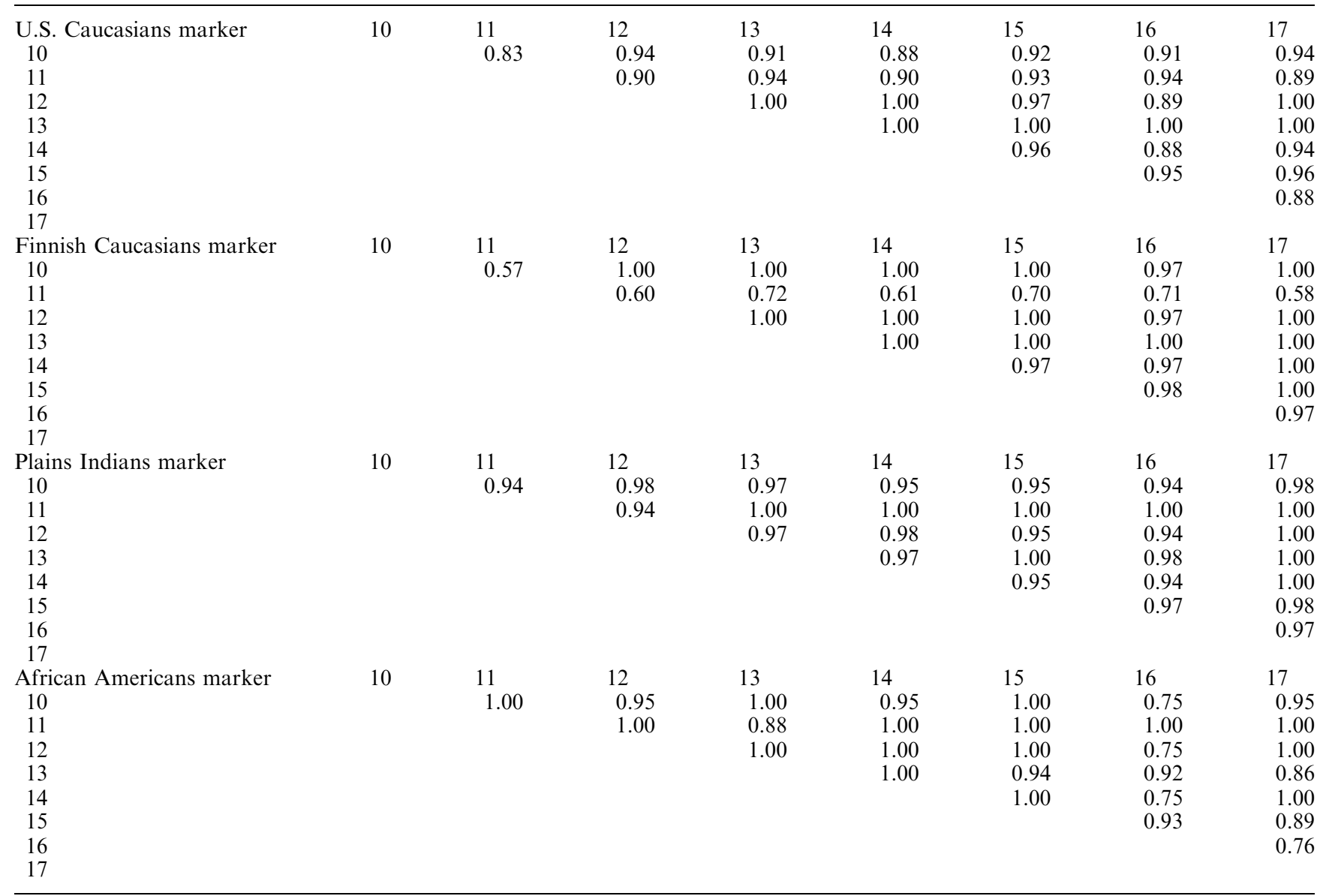

Haplotype analysis Haplotype frequencies were estimated using a Bayesian approach implemented with PHASE (Stephens et al. 2001). These frequencies closely agreed with results from a maximum likelihood method implemented via an expectation-maximization (EM) algorithm (Long et al. 1995).

\section{Results and discussion}

Of a total of $26 S L C 6 A 2 \mathrm{SNPs}, 25$ were polymorphic in all four populations. Dramatic interpopulation differences in allele frequencies were observed for most of the SNPs. Ala457Pro, the previously reported in vitro functional variant, was monomorphic in the 384 individuals representing the four populations. Allele frequencies of SLC6A2 SNPs and their locations in the gene are shown in Table 2. The majority of the markers are located in the intronic sequence of the gene, one synonymous substitution $(\mathrm{A} 1287 \mathrm{G})$ is located in exon 9, one marker is located in the $3^{\prime}$ UTR region, five are in the $3^{\prime}$ region, and one is in the $5^{\prime}$ region (Fig. 1). All genotype frequencies conformed to Hardy-Weinberg equilibrium.
Within SLC6A2, three conserved LD blocks (1, $13.6 \mathrm{~kb} ; 2,12.5 \mathrm{~kb} ; 3,25 \mathrm{~kb}$ ) were observed in all four populations. Definition of haplotype blocks and block boundaries is an inexact science. Some disruptions of $D^{\prime}$ (a measure of LD) occurring within blocks are clearly attributable to low allele frequencies that lead to increased variance in estimation of $D^{\prime}$. We discounted low $D^{\prime}$ values, which appeared to originate from this cause. In the $S L C 6 A 2$ haplotype block regions, $D^{\prime}$ was generally $>0.85$ from one end of the region to the other. $D^{\prime}$ averaged was $0.83,0.94$ and 0.94 in blocks 1,2 , and 3, and perhaps more importantly, the median $D^{\prime}$ value within haplotype blocks was $0.97,0.97$, and 1.00 for blocks 1, 2, and 3, meaning that most of the SNP loci were in very high $\mathrm{LD}$. We note that in the situation that haplotype block boundaries are drawn too widely, an increased number of haplotypes will be observed for the block and an increased number of markers will be required to capture this diversity. SLC6A2 haplotype block boundaries could be drawn somewhat differently than we have done, and it can also be observed that there is some variation from population to population. For example, there was some disruption of LD within block 1 in both Finns and Plains Indians. However, the 
Table 5 Pairwise linkage disequilibrium $\left(D^{\prime}\right)$ among nine single-nucleotide polymorphisms (SNPs) in haplotype block 3 across four populations

\begin{tabular}{|c|c|c|c|c|c|c|c|c|c|}
\hline U.S. Caucasians marker & 18 & 19 & 20 & 21 & 22 & 23 & 24 & 25 & 26 \\
\hline 18 & & 0.97 & 0.97 & 0.97 & 1.00 & 0.89 & 1.00 & 0.97 & 0.96 \\
\hline 19 & & & 1.00 & 1.00 & 1.00 & 1.00 & 1.00 & 1.00 & 0.97 \\
\hline 20 & & & & 1.00 & 1.00 & 1.00 & 1.00 & 1.00 & 1.00 \\
\hline 22 & & & & & & 0.85 & 1.00 & 1.00 & 0.45 \\
\hline 23 & & & & & & & 1.00 & 1.00 & 0.75 \\
\hline 24 & & & & & & & & 1.00 & 1.00 \\
\hline 25 & & & & & & & & & 1.00 \\
\hline \multicolumn{10}{|l|}{26} \\
\hline 18 & & 1.00 & 1.00 & 1.00 & 1.00 & 1.00 & 1.00 & 1.00 & 1.00 \\
\hline 19 & & & 1.00 & 1.00 & 1.00 & 1.00 & 1.00 & 1.00 & 1.00 \\
\hline 20 & & & & 1.00 & 1.00 & 1.00 & 1.00 & 1.00 & 1.00 \\
\hline 21 & & & & & 1.00 & 1.00 & 1.00 & 1.00 & 1.00 \\
\hline 22 & & & & & & 1.00 & 1.00 & 1.00 & 0.86 \\
\hline 23 & & & & & & & 1.00 & 1.00 & 0.76 \\
\hline 19 & & & 1.00 & 1.00 & 1.00 & 1.00 & 1.00 & 1.00 & 0.95 \\
\hline 20 & & & & 1.00 & 1.00 & 1.00 & 1.00 & 1.00 & 0.95 \\
\hline 21 & & & & & 1.00 & 1.00 & 1.00 & 1.00 & 0.95 \\
\hline 22 & & & & & & 1.00 & 1.00 & 1.00 & 1.00 \\
\hline 23 & & & & & & & 1.00 & 1.00 & 1.00 \\
\hline 24 & & & & & & & & 1.00 & 1.00 \\
\hline 25 & & & & & & & & & 0.95 \\
\hline \multicolumn{10}{|l|}{26} \\
\hline African Americans marker & 18 & 19 & 20 & 21 & 22 & 23 & 24 & 25 & 26 \\
\hline 18 & & 1.00 & 1.00 & 1.00 & 0.90 & 0.97 & 0.24 & 1.00 & 0.87 \\
\hline 19 & & & 0.86 & 0.87 & 0.96 & 0.96 & 0.75 & 0.95 & 0.64 \\
\hline 20 & & & & 1.00 & 0.73 & 0.97 & 0.75 & 0.88 & 0.61 \\
\hline 21 & & & & & 0.72 & 0.97 & 0.75 & 0.91 & 0.63 \\
\hline
\end{tabular}

marker panels we genotyped were sufficient to capture diversity in the blocks in the four populations we studied, as described below.

Pairwise LD values within each haplotype block are summarized in Tables 3, 4, and 5; all pairwise LD values among 25 SNPs across four populations are represented in Tables 6, 7, 8, and 9. Haplotype frequencies for the three blocks in four populations are shown in Table 10. For each population and haplotype block, 3-6 common $(\geq 0.05)$ haplotypes accounted for most of the total: 85 $96 \%$ of Caucasian and Plains Indian haplotypes and 75$89 \%$ of African American haplotypes. The number of common $(\geq 0.05)$ haplotypes were block 1: 4, 5, 3, 6; block 2: 5, 5, 4, 5, and block 3: 4, 5, 5, 6 for U.S. and Finnish Caucasians, Plains Indians, and African Americans, respectively.

For each haplotype block, a panel of markers sufficient to maximize genetic information content was available. Excluding Ala457Pro, the number of SNPs available for the three haplotype blocks were 8,8 , and
9 for blocks 1, 2, and 3, respectively. Knowing this, the value of additional SNP markers for haplotype diversity (informativeness) can then be evaluated. We began with the haplotypes derived from all available markers in the block and successively subtracted markers, first subtracting markers that resulted in no change whatever in haplotype diversity and then subtracting markers which changed diversity in the most minimal fashion and so on until we were left with a single, independent, highest heterozygosity SNP marker. The figures graphically depict a reversal of this process, showing that diversity can be maximized using a smaller group of tag SNPs selected from a larger panel. After each marker addition using the pathway determined by the subtraction analysis, haplotype frequencies, and diplotype heterozygosity, the chosen measure of diversity were recalculated. At some point for each haplotype block and each population, the addition of a new SNP marker did not appreciably increase diversity, as shown in Fig. 2. 


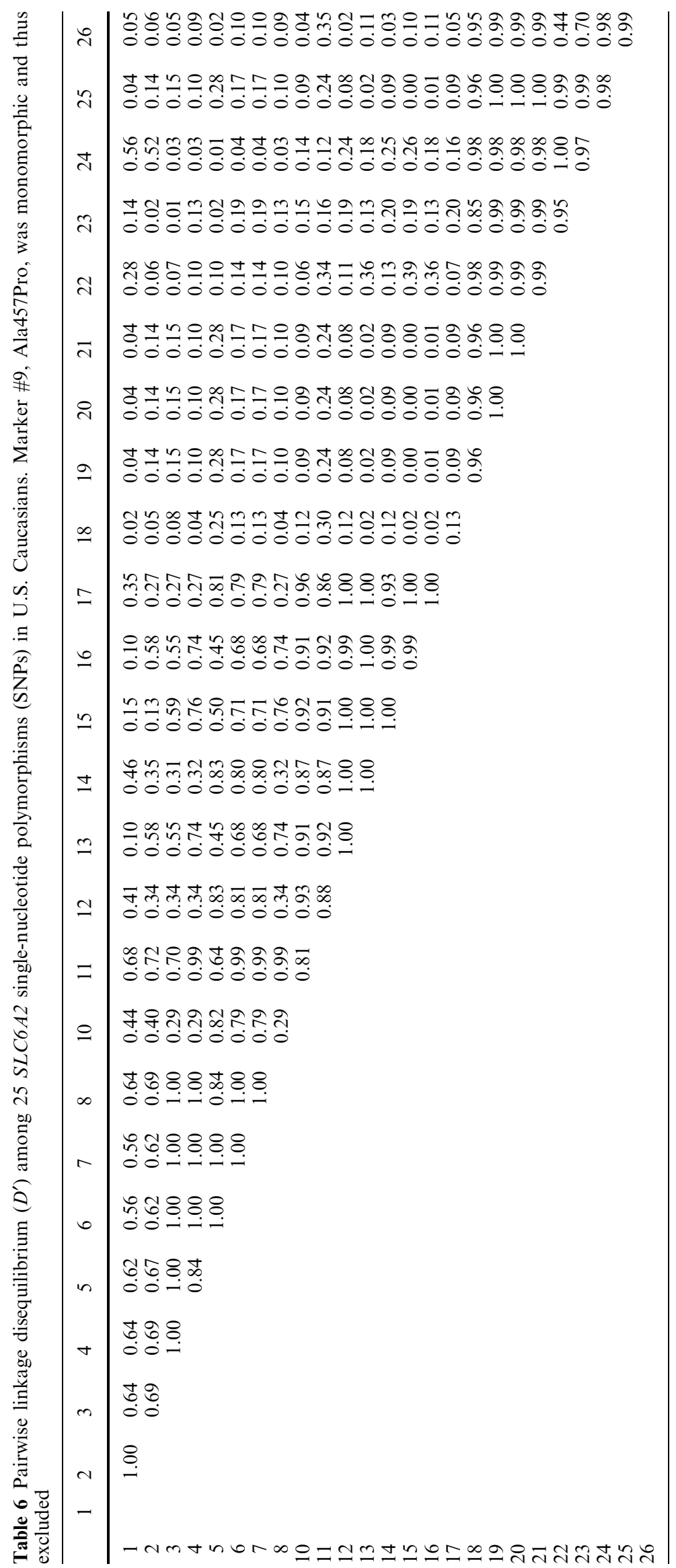




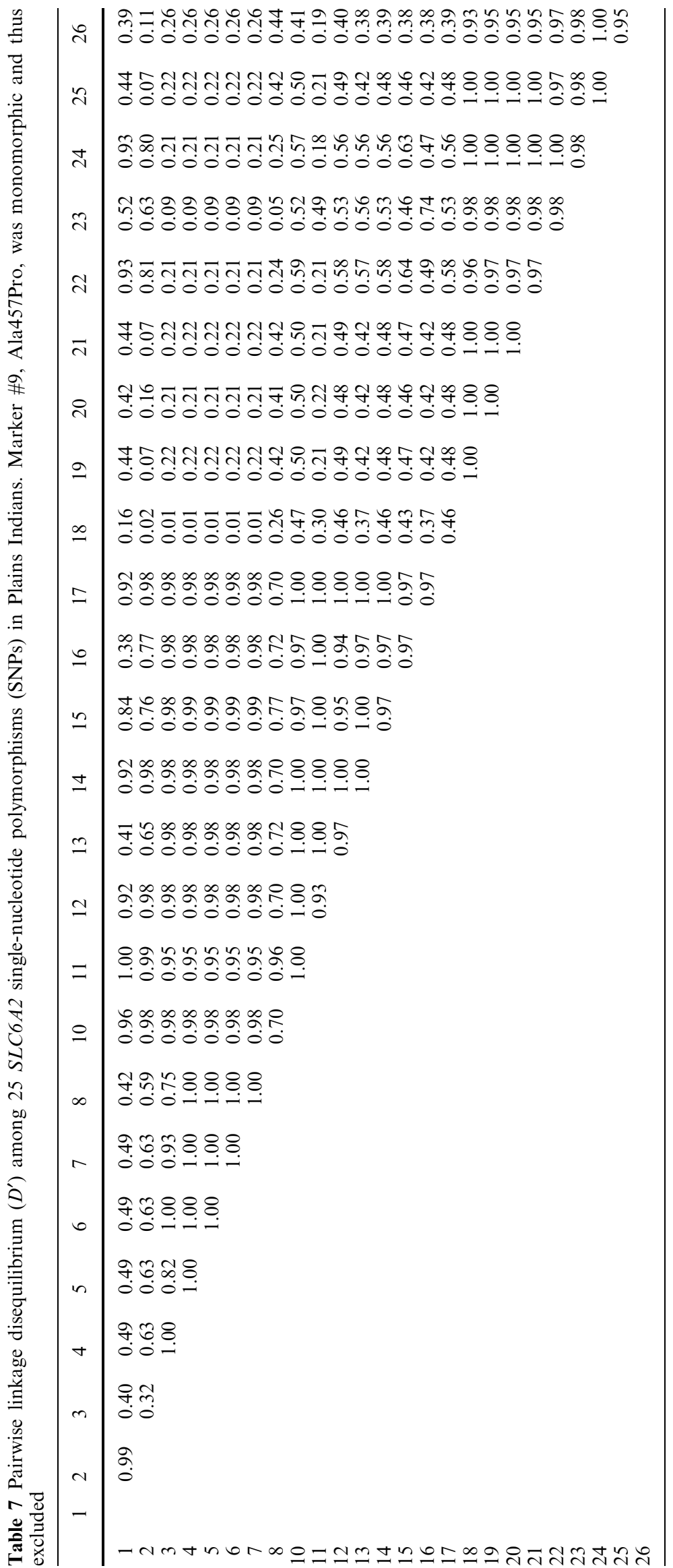




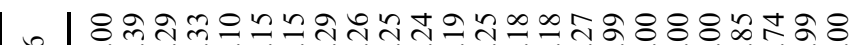

त 00000000000000000-1-100.

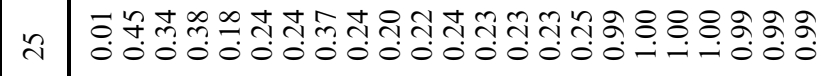

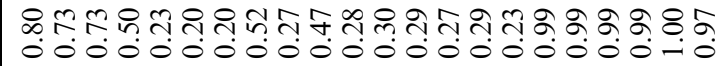

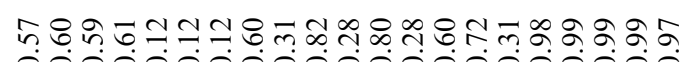
(

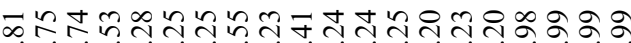

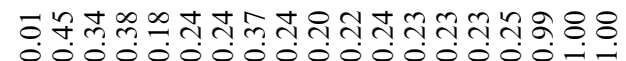

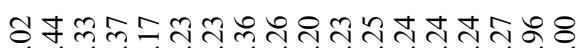

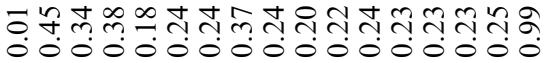

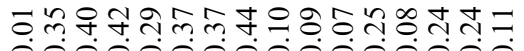

0000000000000000

$=$ =

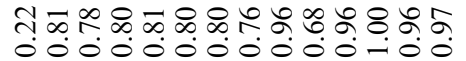

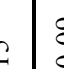

8ำ

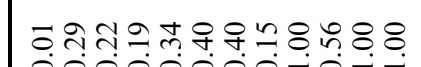

I 0.000.0000.0.

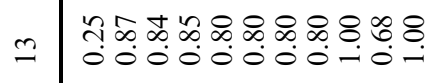

工

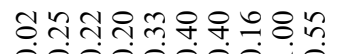

$00000000-10$

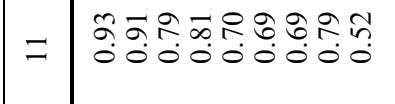

을

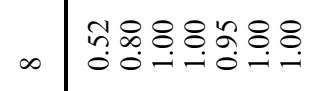

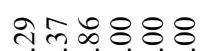

กิกิณะ8

- 0000.

กิกฺุ์

in 0000

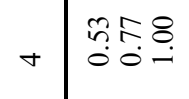

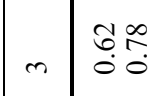

2 8

$-$ 


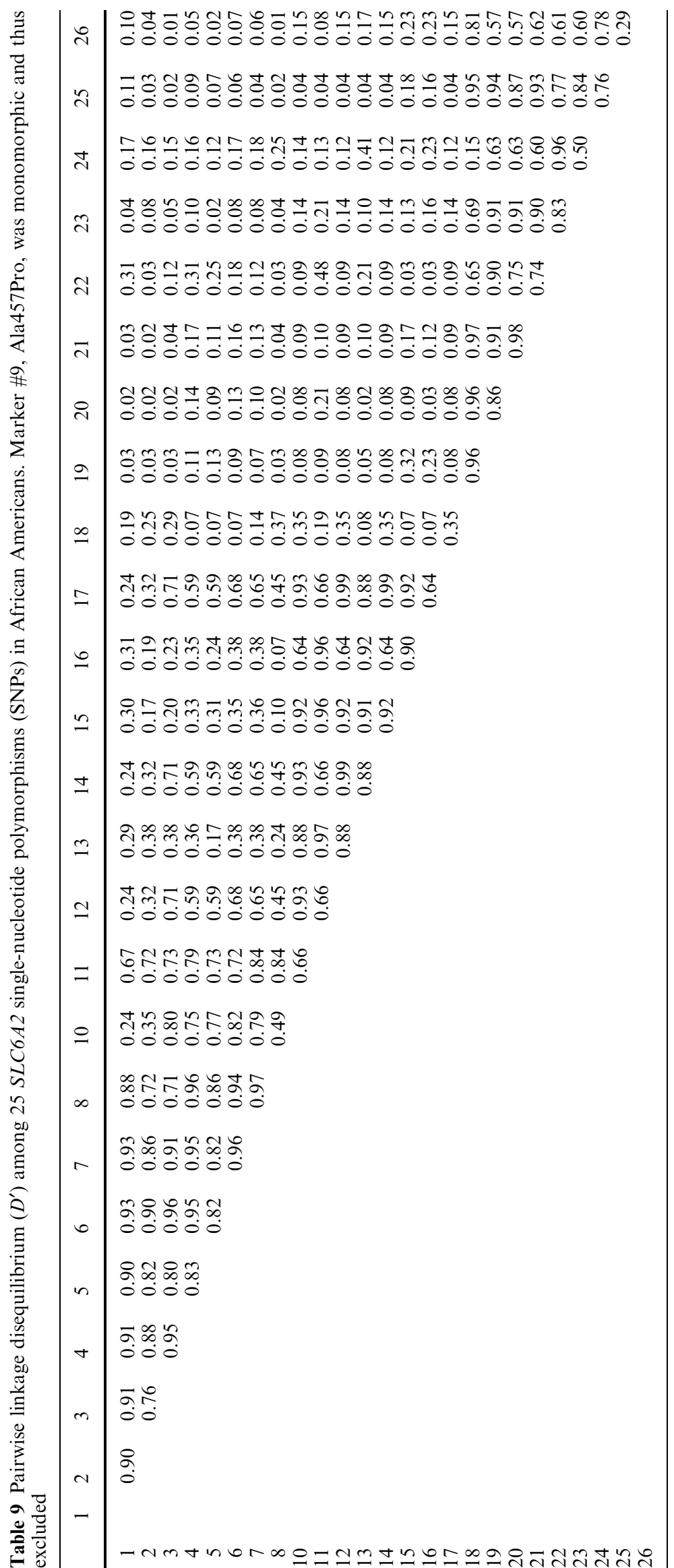


Table 10 Frequencies of haplotypes constructed from 25 single-nucleotide polymorphisms (SNPs) ${ }^{\mathrm{a}}$. $(1=$ allele $1,2=$ allele 2$)$

\section{${ }^{a}$ Haplotypes were constructed using PHASE as described in "Materials and methods" and from 192 chromosomes repre- senting each population}

\begin{tabular}{|c|c|c|c|c|}
\hline & U.S. Caucasians & Finnish Caucasians & Plains Indians & African Americans \\
\hline \multicolumn{5}{|c|}{ Common haplotypes (block 1) } \\
\hline 22212112 & 0.64 & 0.62 & 0.65 & 0.16 \\
\hline 12212112 & 0.11 & 0.08 & 0.21 & 0.2 \\
\hline 11121221 & 0.1 & 0.08 & 0 & 0.26 \\
\hline 11212112 & 0.05 & 0 & 0.1 & 0 \\
\hline 11122111 & 0 & 0.1 & 0 & 0 \\
\hline 22121221 & 0 & 0.06 & 0 & 0 \\
\hline 11112111 & 0 & 0 & 0 & 0.1 \\
\hline 11122221 & 0 & 0 & 0 & 0.06 \\
\hline 12112112 & 0 & 0 & 0 & 0.05 \\
\hline \multicolumn{5}{|c|}{ Common haplotypes (block 2) } \\
\hline 11122212 & 0.35 & 0.29 & 0.2 & 0.12 \\
\hline 22211121 & 0.27 & 0.31 & 0.43 & 0.08 \\
\hline 12112122 & 0.14 & 0.12 & 0 & 0.49 \\
\hline 12122212 & 0.1 & 0.14 & 0.23 & 0.15 \\
\hline 12112222 & 0.05 & 0 & 0.08 & 0 \\
\hline 21211121 & 0 & 0.07 & 0 & 0 \\
\hline 12112212 & 0 & 0 & 0 & 0.05 \\
\hline \multicolumn{5}{|c|}{ Common haplotypes (block 3) } \\
\hline 112121221 & 0.31 & 0.25 & 0.28 & 0.29 \\
\hline 221221212 & 0.24 & 0.33 & 0.35 & 0.07 \\
\hline 112122221 & 0.17 & 0.14 & 0.08 & 0.04 \\
\hline 112111121 & 0.13 & 0.16 & 0.15 & 0.04 \\
\hline 121221212 & 0.04 & 0.08 & 0.08 & 0.11 \\
\hline 112111221 & 0 & 0 & 0 & 0.08 \\
\hline 112122222 & 0 & 0 & 0 & 0.13 \\
\hline 112121211 & 0 & 0 & 0 & 0.07 \\
\hline
\end{tabular}

The required number of tag SNPs varies according to the haplotype diversity of the region (and population) and the information content of the markers available. Haplotype diversity in block 1 was greatest in African Americans, and to maximize it, more markers were needed (5-6 markers). For the other populations, 2-3 markers sufficed for block 1, but the optimal markers differed across populations. Thus, each SNP had different information content in different populations. For association/linkage studies, different tag SNPs could be used in different target populations. Alternatively, the entire panel of 25 SNPs could be applied to reliably capture haplotype diversity across populations. As illustrated in Fig. 2, genotyping larger panels of markers yields a steadily diminishing return, but another purpose of this approach is to capture more information on certain rarer haplotypes. The focus of haplotype-based genetic association studies has been the detection of effects of moderately abundant loci, because haplotypes and functional alleles of low frequency are not well represented in small datasets. However, power increases in larger datasets that may be available for certain noradrenergic related phenotypes, for example, diseases such as hypertension and mood disorders, which are readily diagnosed and which afflict very large segments of populations.
For SLC6A2, the 25-locus SNP panel defines a three-block LD structure across the entire gene region and would be sufficient to capture the signal of any moderately abundant SNP. For example, A1287G is the marker most extensively used in NET linkage studies, and this SNP is located in block 2, for which the SLC6A2 SNP panel includes another seven markers in addition to A1287G. When A1287G is excluded, $99 \%$ of the information content of block 2 is still captured.

In conclusion, the SLC6A2haplotype map and marker panel are a comprehensive tool for genetic linkage studies on phenotypes related to noradrenergic function. This map is a surrogate for moderately abundant effective alleles, which may be unknown or unrecognized as functional.

Acknowledgements We are grateful to Dr. Alec Roy and Dr. Matti Virkkunen for subsets of their population datasets, to Longina Akhtar for assistance with cell culture, and Ilona Lorincz for technical assistance.

Supported by NIDCR Intramural Grant Z01, NIAAA Intramural Grant Z01 AA000301 (National Institutes of Health, Bethesda, MD, USA), and the Comprehensive Neuroscience Program Grant USUHS G192BR-C4 (Henry Jackson Foundation, Rockville, MD, USA). 
Fig. 2a-c Effect of successively adding optimal SNPs on haplotype diversity in four populations. The successive addition of SNPs determined by the subtraction path, which maximally retained haplotype diversity, identifies a minimal set of tag SNPs to maximize diversity (diplotype heterozygosity) within SLC6A2 haplotype blocks $(\mathbf{a}-\mathbf{c})$ in four populations
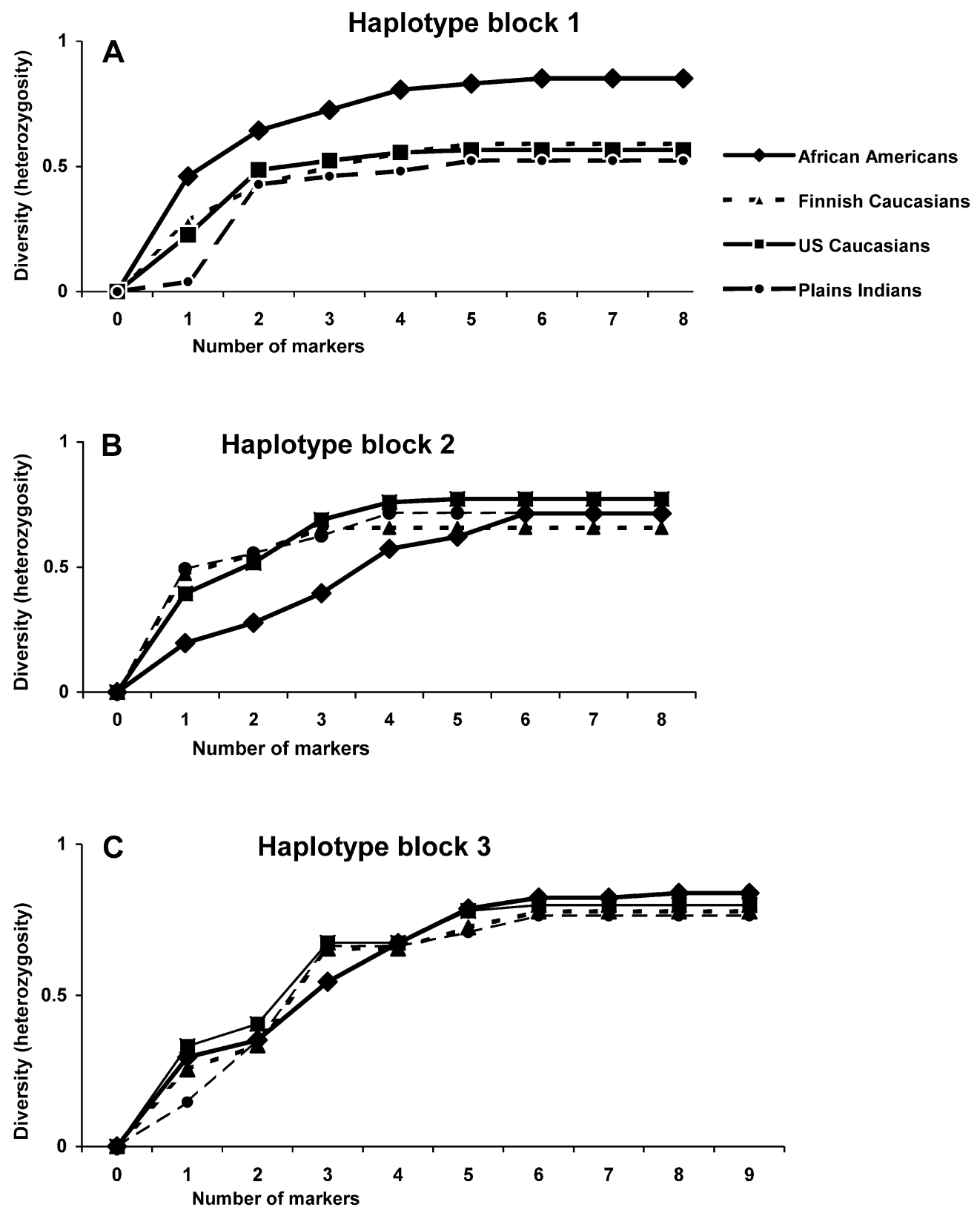

\section{References}

Backs J, Haunstetter A, Gerber SH, Metz J, Borst MM, Strasser RH, Kubler W, Haass M (2001) The neuronal norepinephrine transporter in experimental heart failure: evidence for a posttranscriptional downregulation. J Mol Cell Cardiol 33:461-472

Bohm M, La Rosee K, Schwinger RH, Erdmann E (1995) Evidence for reduction of norepinephrine uptake sites in the failing human heart. J Am Coll Cardiol 25:146-153

Bohm M, Castellano M, Flesch M, Maack C, Moll M, Paul M, Schiffer F, Zolk O (1998) Chamber-specific alterations of norepinephrine uptake sites in cardiac hypertrophy. Hypertension 32:831-837

Brüss M, Kunz J, Lingen B, Bönisch H (1993) Chromosomal mapping of the human gene for the tricyclic antidepressantsensitive noradrenaline transporter. Hum Genet 91:278-280

Bymaster FP, Katner JS, Nelson DL, Hemrick-Luecke SK, Threlkeld PG, Heiligenstein JH, Morin SM, Gehlert DR, Perry
KW (2002) Atomoxetine increases extracellular levels of norepinephrine and dopamine in prefrontal cortex of rat: a potential mechanism for efficacy in attention deficit/hyperactivity disorder. Neuropsychopharmacology 27:699-711

Esler M, Jackman G, Bobik A, Leonard P, Kelleher D, Skews H, Jennings G, Korner P (1981) Norepinephrine kinetics in essential hypertension. Defective neuronal uptake of norepinephrine in some patients. Hypertension 3:149-156

Foote SL, Bloom FE, Aston-Jones G (1983) Nucleus locus ceruleus: new evidence of anatomical and physiological specificity. Physiol Rev 63:844-914

Gabriel SB, Schaffner SF, Nguyen H, Moore JM, Roy J, Blumenstiel B, Higgins J, DeFelice M, Lochner A, Faggart M, LiuCordero SN, Rotimi C, Adeyemo A, Cooper R, Ward R, Lander ES, Daly MJ, Altshuler D (2002) The structure of haplotype blocks in the human genome. Science 296:2225-2229

Gu HH, Wall S, Rudnick G (1996) Ion coupling stoichiometry for the norepinephrine transporter in membrane vesicles from stably transfected cells. J Biol Chem 271:6911-6919 
Hahn MK, Blakely RD (2002) Gene organization and polymorphisms of monoamine transporters. Relationship to psychiatric and other complex diseases. In: Reith MEA (ed) Neurotransmitter transporters. Structure, function, and regulation, Humana, Totowa, NJ, pp 111-169

Hahn MK, Robertson D, Blakely RD (2003) A mutation in the human norepinephrine transporter gene (SLC6A2) associated with orthostatic intolerance disrupts surface expression of mutant and wild-type transporters. J Neurosci 23:4470-4478

Ivancsits S, Heider A, Rudiger HW, Winker R (2003) Orthostatic intolerance is not necessarily related to a specific mutation (Ala457Pro) in the human norepinephrine transporter gene. Am J Med Sci 325:63-65

Iversen LL (1974) Uptake mechanisms for neurotransmitter amines. Biochem Pharmacol 23:1927-1935

Jasmin L, Tien D, Weinshenker D, Palmiter RD, Green PG, Janni G, Ohara PT (2002) The NK1 receptor mediates both the hyperalgesia and the resistance to morphine in mice lacking noradrenaline. Proc Natl Acad Sci 99:1029-1034

Jordan S, Kramer GL, Zukar PK, Moeller M, Petty F (1994) In vivo biogenic amine efflux in medial prefrontal cortex with imipramine, fluoxetine, and fluvoxamine. Synapse 18:294-297

Leszczynska-Rodziewicz A, Czerski PM, Kapelski P, Godlewski S, Dmitrzak-Weglarz M, Rybakowski J, Hauser J (2002) A polymorphism of the norepinephrine transporter gene in bipolar disorder and schizophrenia: lack of association. Neuropsychobiology 45:182-185

Long JC, Williams RC, Urbanek M (1995) An E-M algorithm and testing strategy for multiple locus haplotypes. Am J Hum Genet 56:799-810

Merlet P, Dubois-Rande JL, Adnot S, Bourguignon MH, Benvenuti C, Loisance D, Valette H, Castaigne A, Syrota A (1992) Myocardial beta-adrenergic desensitization and neuronal norepinephrine uptake function in idiopathic dilated cardiomyopathy. J Cardiovasc Pharmacol 19:10-16

Nelson N (1998) The family of $\mathrm{Na}^{+} / \mathrm{Cl}^{-}$neurotransmitter transporters. J Neurochem 71:1785-1803

Pacholczyk T, Blakely RD, Amara SG (1991) Expression cloning of a cocaine- and antidepressant-sensitive human noradrenaline transporter. Nature 350:350-354

Pörzgen P, Bönisch H, Brüss M (1995) Molecular cloning and organization of the coding region of the human norepinephrine transporter gene. Biochem Biophys Res Commun 215:11451150

Pörzgen P, Bönisch H, Hammermann R, Brüss M (1998) The human noradrenaline transporter gene contains multiple polyadenylation sites and two alternatively spliced C-terminal exons. Biochim Biophys Acta 1398:365-370
Ritz MC, Cone EJ, Kuhar MJ (1990) Cocaine inhibition of ligand binding at dopamine, norepinephrine and serotonin transporters: a structure-activity study. Life Sci 46:635-645

Sacchetti G, Bernini M, Bianchetti A, Parini S, Invernizzi RW, Samanin R (1999) Studies on the acute and chronic effects of reboxetine on extracellular noradrenaline and other monoamines in the rat brain. Br J Pharmacol 128:1332-1338

Samochowiec J, Kucharska-Mazur J, Kaminski R, Smolka M, Rommelschpacher H, Wernicke C, Tymicz A, Schmidt LG (2002) Norepinephrine transporter gene polymorphism is not associated with susceptibility to alcohol dependence. Psychiatry Res 111:229-233

Schnell O, Muhr D, Weiss M, Dresel S, Haslbeck M, Standl E (1996) Reduced myocardial 123I-metaiodobenzylguanidine uptake in newly diagnosed IDDM patients. Diabetes 45:801805

Schomig E, Fischer P, Schonfeld CL, Trendelenburg U (1989) The extent of neuronal re-uptake of $3 \mathrm{H}$-noradrenaline in isolated vasa deferentia and atria of the rat. Arch Pharmacol 340:502508

Shi MM, Myrand SP, Bleavins MR, de la Iglesia FA (1999) High throughput genotyping for the detection of a single nucleotide polymorphism in NAD $(\mathrm{P}) \mathrm{H}$ quinone oxidoreductase (DT diaphorase) using TaqMan probes. Mol Pathol 52:295-299

Stephens M, Smith NJ, Donnelly P (2001) A new statistical method for haplotype reconstruction from population data. Am J Hum Genet 68:978-989

Stober G, Nothen MM, Porzgen P, Bruss M, Bonisch H, Knapp M, Beckmann H, Propping P (1996) Systematic search for variation in the human norepinephrine transporter gene: identification of five naturally occurring missense mutations and study of association with major psychiatric disorders. Am J Med Genet 67:523-532

Tanda G, Pontier FE, Frau R, DiChiara G (1997) Contribution of blockade of the noradrenaline carrier to the increase of extracellular dopamine in the rat prefrontal cortex by amphetamine and cocaine. Eur J Neurosci 9:2077-2085

Tatsumi M, Groshan K, Blakely RD, Richelson E (1997) Pharmacological profile of antidepressants and related compounds at human monoamine transporters. Eur J Pharmacol 340:249258

Zahniser NR, Doolen S (2001) Chronic and acute regulation of $\mathrm{Na}^{+} / \mathrm{Cl}^{-}$-dependent neurotransmitter transporters: drugs, substrates, presynaptic receptors, and signaling systems. Pharmacol Ther 92:21-55 